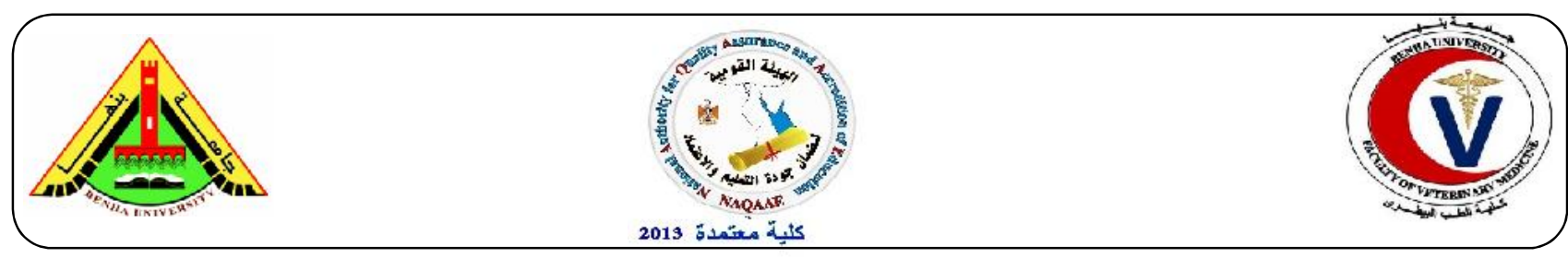

\title{
Effect of chitosan and calcium phosphate nanoparticles on PPRV vaccine immunogenicity and keeping quality
}

\author{
Gabr. F. El-Bagoury ${ }^{1}$, Ehab. M. EL-Nahas ${ }^{1}$, Ahmed Husien Mostafa ${ }^{2}$, Mohamed abd el bar saafan ${ }^{2}$ \\ ${ }^{1}$ Department of virology, Faculty of Veterinary Medicine, Benha University \\ ${ }^{2}$ Veterinary serum and vaccine Research institute, Abbasia, cairo.
}

\section{A B S T R A C T}

Nanotechnology that created nanoparticles (NP) having superior properties compared to the original ones and offers a new era for vaccine improvement. In this study, Chitosan nanoparticle (CSN) and Calcium Phosphate nanoparticle $(\mathrm{CaPN})$ based-vaccines were prepared in different concentrations $[1 \%(\mathrm{~W} / \mathrm{V}) \& 0.125 \%(\mathrm{~W} / \mathrm{V})]$ then, trials to discover the effects of these NP based-Pest des Petits Ruminants (PPR) vaccines on the immunogenicity and keeping quality compared than that offered by the conventional PPR vaccine. The results revealed from the serum neutralization test (SNT) that performed on sera collected from inoculated sheep with NP based vaccines and conventional one on a weekly base interval for four weeks and with the same subcutaneously inoculated dose of $10^{3}$ TCID $_{50}$ showed that all NP based vaccines was faster in yielding neutralizing antibody titer from week one while conventional vaccine was delayed to the second week, meanwhile CSN $0.125 \%$ based vaccine was more superior than other NP based vaccines that reached the peak titer [Geometric Mean Titer (GMT) of 128] by the fourth week while conventional vaccine results were only showed 53.3 as GMT. These results confirmed that $\mathrm{CSN}$ and $\mathrm{CaPN}$ based vaccines were generally more superior than conventional one and CSN $0.125 \%$ based vaccine were achieved the forefront results. On the other hand, keeping quality tests based upon PPR virus titration of all types of vaccines in this trial that were exposed to different temperature degrees $\left(4^{\circ} \mathrm{c}, 25^{\circ} \mathrm{c} \& 37^{\circ} \mathrm{c}\right)$ were also studied. The obtained results emphasized that $\mathrm{CSN} 0.125 \%$ based vaccine superiority that showed only $0.4 \log$ $1_{10 \mathrm{TCID}} / \mathrm{ml}$, as a titer loss after lyophilization process, while the conventional vaccine lost $0.6 \log$ $10 \mathrm{TCID}_{50} / \mathrm{ml}$.On keeping all types of vaccines employed in this study at $4^{\circ} \mathrm{c}$ (for 12 weeks), $25^{\circ} \mathrm{c}$ (for 12 weeks) and $37^{\circ} \mathrm{c}$ (for 7 days), it was found that CSN $0.125 \%$ based vaccine showed the lowest loss in titer among other NP based vaccines in our study, that was expressed by $\log$ $10 \mathrm{TCID}_{50} / \mathrm{ml}$ as $0.4,1.9$ and 1.4 respectively. While loss of titer in case of conventional vaccine that kept at the same degrees of temperature was 0.5, 2.2 and 1.6 respectively. So, these results favored CSN $0.125 \%$ based vaccine that showed better results in immunogenicity and keeping quality rather than conventional PPR vaccine.

Keywords: Chitosan nanoparticle, Calcium Phosphate nanoparticle, PPRV vaccine, immunogenicity and keeping quality. 


\section{INTRODUCTION}

Vaccine formulations including nanoparticles (NP) and antigens can be classified by NP action into those based on delivery system or immune potentiators (Mody et al., 2013). Microparticles covalently coupled with antigen offer distinct advantages namely, a low dose of antigen is required, efficient processing by antigen-presenting cells and stability during storage (Gengoux and Leclerc 1995). Role of NP in drug delivery is well known. The most exciting aspects of NP which make them a special class of nano medicine and now in vaccinology are the ability to co-deliver antigen and immune potentiator (Fujita et al., 2011).

Several opportunities of NP as a suitable adjuvant for vaccine development, still challenges exist. In the beginning, efforts were made to encapsulate the protein antigen inside particle, but harsh manufacturing process resulted degradation of antigen and poor immunogenicity (Jain et al, 2011). NP adjuvants increase the immunogenicity of a vaccine by mimicking pathogen-associated molecular patterns they can activate pattern recognition receptors, such as Toll-like receptors and trigger intracellular signaling cascades that initiate the innate immune response, resulting in enhancement of the adaptive immune response, also NP up regulating co-stimulatory molecules on antigen presenting cells, they act as a store to provide prolonged delivery of antigens. (Nordly et al. 2009). The primary mechanisms of NP adjuvant function are stabilization of antigen, delivery of antigen and activation of innate immunity (Akagi et al., 2012).

Chitosan nanoparticles (CSN) have been synthesized as drug and vaccine delivery carriers as reported in previous studies (van der Lubben et al. 2001and De Campos et al., 2001). Due to their bioadhesive, biocompatibility, biodegradability and penetration-enhancement properties, CSN are most efficiently taken up by phagocytotic cells inducing strong systemic and mucosal immune responses against antigens (Illum et al., 2001, Zhu et al., 2007).CSN had a strong potential to increase both cellular and humoral immune responses and elicited a balanced ( $\mathrm{T}$-helper 1)Th1/Th2(T-helper 2) response, and that CSN may be a safe and efficacious adjuvant candidate suitable for a wide spectrum of prophylactic and therapeutic vaccines (ZhengShun Wen et al., 2011).

Calcium phosphate nanoparticles $(\mathrm{CaPN})$ as a biodegradable NP have revealed a new highly interesting and novel platform for adjuvant development and designing being safer than alum-based adjuvant systems. Also, $\mathrm{CaPN}$ did not elicit IgE response. (He et al., 2000). CaPN of a particles of size 50-100 nm diameters provide safe and easily manufactured vaccine adjuvant and delivery as some trials done for Foot and Mouth Disease virus (Joyappa et al., 2009).

Live attenuated vaccines on exposing to a temperature of $4^{\circ} \mathrm{c}$, it will be damaged after 30 days only, while after keeping at a higher temperature of $37^{\circ} \mathrm{c}$ it will be lost just after 10 hours (Sarkar et al., 2003). Riyesh (2011) also added that the longest time that can spent before live attenuated PPR vaccine being destroyed is 7 days at a temperature of $37^{\circ} \mathrm{C}$.

\section{Aim of the work}

This study aimed to assess both of improving the immunogenicity and the keeping quality of different vaccine types (conventional and nano-based).

\section{Materials and Methods}

\subsection{PPR Virus:}

The Nig/75 strain of PPRV (with a titer of $10^{6}$ TCID 50/ml) was provided from Rinderpest virus research dept., vet. serum and vaccine research institute, Cairo.

Used for virus titration. 


\subsection{Patent (Conventional) PPR Vaccine:}

A living attenuated PPR tissue culture patented vaccine (each dose contains $10^{3}$ TCID 50/dose). It was provided from Rinderpest virus research dept., vet. serum and vaccine research institute, Cairo. It was issued as a lyophilized material and prepared from the Nigerian strain (Nigeria 75/1). It should be reconstituted in sterile chilled physiological saline for a subcutaneous injection.

Used for virus titration.

\subsection{VERO cells (Green monkey kidney cells):}

It was maintained and grown in Eagles minimum essential media supplemented with $10 \%$ newly born calf serum, antifungal, penicillin sodium $100 \mathrm{IU} / \mathrm{ml}$ and streptomycin $100 \mathrm{mg} / \mathrm{ml}$. It was used in nanoparticles-based vaccines preparation, virus titration, serum neutralization test (SNT).

Obtained from VACCERA, Giza, Egypt.

\subsection{Virus titration:}

The virus titration of the prepared virus fluids was performed using the 10-fold dilution inoculated onto VERO cells microtiter plate according to OIE (2010) and the virus titer was calculated following the rules given by Reed and Muench (1938).

Virus titration were done just prior to lyophilization using PPRV infected fluid and later on further titrations were done using lyophilized vaccine vials during keeping quality tests.

\subsection{Experimental nanoparticles-based vaccines preparation:}

\subsubsection{Chitosan nanoparticles stock solution} preparation:

Chitosan nanoparticles preparations were performed by dissolved chitosan at $5 \%(\mathrm{w} / \mathrm{v})$ with $1 \%(\mathrm{w} / \mathrm{v})$ acetic acid (HOAc) then $\mathrm{pH}$ adjusted to $4.6-4.8$ with $10 \mathrm{~N} \mathrm{NaOH}$. CSN were formed by the principle of ionic crosslinking between positively charged chitosan and negatively charged sodium tripolyphosphate (TPP) $(0.25 \% \mathrm{w} / \mathrm{v})$ according to Zhao et al., (2012). Chitosan nanoparticles were formed by dropping TPP to the chitosan solution with the ratio of (1:5) with magnetic stirring at room temperature for overnight. CSN was separated by centrifugation at 10,000 rpm for 30 minutes at $4{ }^{\circ} \mathrm{C}$ and the supernatant was discarded while the sediment was reconstituted to the original volume with PBS, So, a stock CSN suspension with a concentration of $3 \mathrm{mg} / \mathrm{ml}$ was obtained. CSN suspension was added to MEM with ratios of $1 \%(30 \mathrm{ug} / \mathrm{ml}), 0.125 \%(3.75 \mathrm{ug} / \mathrm{ml})$ to obtain working solutions CSN-1\% and CSN-0.125\% Qi et al.,( 2004).Chitosan was obtained from Naquaa foundation, Giza, Egypt.

\subsubsection{Calcium phosphate nanoparticles stock solution preparation:}

Calcium phosphate $(\mathrm{CaPN})$ is amorphous nano-powder, < $150 \mathrm{~nm}$ particle size. It was obtained from Sigma Aldrich and prepared by dissolving in deionized water to make $10 \%$ stock and the solution subjected to continuous stirring for 6 hours at room temperature, followed by sonication for three times repeated cycles each of 15 minutes, according to Saeed et al., (2015). Then, the obtained $10 \%$ stock solution was further diluted till obtaining $\mathrm{CaPN}-1 \%$ and CaPN- $0.125 \%$ working solutions.

\subsubsection{Propagation of PPRV on Vero cells:}

It was performed through infecting actively growing Vero cells suspended in complete culture medium, with the working seed PPRV at MOI of $0.01 \mathrm{TCID}_{50}$ per cell. Cell culture flasks are filled with this virus/cell mixture (around $2 \times 10^{7}$ vero cells in a $175 \mathrm{~cm}^{3}$ flask), and are incubated at $37{ }^{\circ} \mathrm{C}$. The cultured cells are examined regularly to detect a CPE. The medium (nanoparticles working solution) is renewed every 2 days, reducing the proportion of serum to $2 \%$ once the cell monolayer is complete. Virus is first harvested when there is $40-50 \%$ CPE. This viral suspension is stored at $-70^{\circ} \mathrm{C}$. Successive harvesting is carried out every 2 days until the CPE reaches $70-80 \%$ 
which is the time for final freezing of the culture flasks. All suspensions of virus collected are submitted to two freeze-thaw cycles, and then added to form a single batch.

\subsubsection{Stabilization of Viral Suspension:}

Weybridge medium (WBM) was composed of an aqueous mixture of $2.5 \%$ lactalbumin hydrolysate, $5 \%$ sucrose and $1 \%$ monosodium salt of glutamic acid.

The stabilizers were used as a volumetrically equal amount to the PPRV-suspension, mixed together just prior to lyophilization.

\subsubsection{Lyophilization (freeze drying):}

Performed in accordance with (Wang and Zhang 2007, Zhou et al., 2007 and Shao-zhi et al.,2010) as

\subsubsection{Quality control tests of nanoparticles based PPR vaccines:}

2.5.6.1 Sterility test for confirmation of the purity for the obtained vaccine from aerobic and anaerobic bacteria and other viruses where nutrient broth, thioglycolate media, PPLO media were used according to the USA code of federal regulation CFR (1987).

2.5.6.2 Safety test for detection of nonspecific toxicity after vaccines inoculation in laboratory animals (guineapigs and mice) was performed in accordance with OIE (2013).

2.5.6.3 Identity test for confirmation of the presence of PPR virus using a reference hyperimmune serum that was obtained from Department of the rinderpest, VSVRI, Abbassia, Cairo according guidelines reported by OIE (2013).

\subsection{Lyophilization (freeze drying) of live attenuated stabilized PPRV vaccines:}

The lyophilizing technique was carried out on Tofflon lyophilizer apparatus according to (Wang and Zhang 2007). The shelf temperature was set at $-32{ }^{\circ} \mathrm{C}$, and the vacuum was controlled under $10 \mathrm{~Pa}$ (Zhou et al., 2007). After freeze-drying, the vials were sealed and kept at room temperature for 2 hours (Shao-zhi et al.,2010) and then kept at $-20^{\circ} \mathrm{c}$ till subjected for evaluation of the effect of lyophilization process.

2.7. Thermostability of lyophilized live attenuated PPRV vaccine:

Ten lyophilized vaccine vials for each vaccine type (Conventional/Nanoparticles-based) were stored at $4{ }^{\circ} \mathrm{C} \& 25^{\circ}$ (room temperature) up to 12 weeks. Samples were taken in two weeks intervals and the lyophilized samples were reconstituted with $1 \mathrm{ml}$ of distilled water and subjected for PPRV titration.

Also, 10 vials of lyophilized vaccine types were kept in the incubator $\left(37^{\circ} \mathrm{C}\right)$ up to 7 days. Samples were taken in two days intervals and the lyophilized samples were reconstituted with $1 \mathrm{ml}$ of distilled water and subjected for PPRV titration.

\subsection{Animals and experimental design:}

Eighteen local breed sheep of nine to twelve months old were used. These Sheep were apparently healthy and free from antibodies against PPR virus as proved by using serum neutralization test (SNT). The sheep were used to compare the efficacy of the different types of vaccines by dividing into six groups as follow:

Group (1): Animals vaccinated with PPR vaccine based on $0.125 \%$ Calcium phosphate nanoparticles (P-0.125\% coded vaccine).

Group (2): Animals vaccinated with PPR vaccine based on $1 \%$ Calcium phosphate nanoparticles (P-1\% coded vaccine).

Group (3): Animals vaccinated with PPR vaccine based on $0.125 \%$ Chitosan nanoparticles (S-0.125\% coded vaccine).

Group (4): Animals vaccinated with PPR vaccine based on $1 \%$ Chitosan nanoparticles (S-1\% coded vaccine).

Group (5): Animals vaccinated with PPRV conventional type vaccine (positive control) $(\mathbf{C}$ coded vaccine).

Group (6): Unvaccinated control animals (negative control).

Lyophilized vial of each previously mentioned vaccine type was reconstituted in $100 \mathrm{ml}$ sterile 
chilled physiological saline for a subcutaneous injection by $1 \mathrm{ml}$ (containing 103 TCID $_{50}$ ) per a sheep.

\subsection{Serum samples:}

Serum samples were collected from vaccinated and unvaccinated sheep on the day of vaccination (zero day), then weekly till 28th day post vaccination for serological investigation. The sera were inactivated at $56^{\circ} \mathrm{C}$ for 30 minutes, and then stored at $-20^{\circ} \mathrm{C}$ until used in Serum Neutralization Test (SNT) for detection of specific antibodies for PPRV. The vaccinated and unvaccinated control animals were observed clinically during the experiment for any clinical abnormalities during the experiment.

\subsection{Serum neutralization test OIE (2013):} According to OIE recommendations in 2013.This test was carried out for both qualitatively to screen animals sera samples to prove their susceptibility, as well as quantitatively to estimate the neutralizing antibody titers in animals sera, acquired through PPRV vaccine inoculations.

\subsection{Reference hyper-immune serum:}

Reference hyper-immune serum against PPRV were obtained from Department of the rinderpest, VSVRI, Abbassia, Cairo. It was used in PPRV identity, SNT and immunogenicity testing.

\section{RESULTS}

\subsection{Evaluating the quality control tests of nanoparticles based PPR vaccines:}

Sterility tests reflected the purity of different nanoparticles based PPR vaccines from any bacterial contaminants either aerobic or nonaerobic one. However, safety profile of all types of prepared vaccines was confirmed as during 3 weeks of quality control observation, none of the laboratory animals either guineapigs or mice showed any harmful effects and was found in a good health. Also, identity test confirmed the presence of PPRV that neutralized by using a specific hyper-immune serum.

\subsection{Evaluating the immunogenicity of PPR vaccines using SNT:}

Referring to table (1) and chart (1) that showed the PPRV neutralizing antibody titers, the following facts were clearly noticed that the fastest onset of appearance of the neutralizing antibody titers was in group 3 (S- $0.125 \%$ Vaccine) with GMT $=10.6$ by the end of the first week. that consider protective. However, the highest results obtained by the end of 4th; week also, observed in group 3.

(S- $0.125 \%$ Vaccine) with GMT $=128$. Meanwhile, the lower the concentration of nanoparticles based- vaccines, the higher the neutralizing antibody titers. Also, all nanoparticle -based vaccines (group 1,2,3\&4) yielded too much better result rather than the conventional vaccine (group 5).

\subsection{Keeping Quality tests of PPR vaccines:}

Referring to table (2), although it was well known that lyophilization causes loss of PPRV titer by about $0.6 \log 10 \mathrm{TCID}_{50} / \mathrm{ml}$ (as done by conventional vaccine), but $\mathrm{S}-0.125 \%$ vaccines lost only $0.4 \log 10 \mathrm{TCID}_{50} / \mathrm{ml}$. also, almost other nanoparticles-based vaccines in other groups showed lower loss compared with that done by conventional one.

Table (3) showed that the lowest loss of efficacy among all types of vaccines was in S$0.125 \%$ Vaccine $(0.4 \log 10)$ while the highest loss was in P-1\% Vaccine (0.6 log 10), However the conventional vaccine lost ( $0.5 \mathrm{log}$ 10 ), Loss of efficacy of the vaccine was calculated by the difference between the staring log (just after lyophilization) and the ending $\log$ (at week no. 12).

Table (4) showed that the lowest loss of efficacy among all types of vaccines was in S$0.125 \%$ Vaccine (1.9 log 10) while the highest loss was in S-1\% Vaccine (2.2 log 10). The same as the conventional vaccine lost (2.2 log 
10), Loss of efficacy of the vaccine was calculated by the difference between the staring log (just after lyophilization) and the ending $\log$ (at week no. 12).

Table (5) showed that the lowest loss of efficacy among all types of vaccines was in
S-0.125\% Vaccine (1.4 $\log 10)$ while the highest loss was in S-1\% Vaccine (1.6 log 10 ).The same as the conventional vaccine lost (1.6 $\log 10)$, Loss of efficacy of the vaccine was calculated by the difference between the staring $\log$ (just after lyophilization) and the ending $\log$ (at day no. 7).

Table 1. Assessment of CSN-based PPR and CaPN -based PPR vaccines on the immune response of susceptible sheep comparing with conventional one.

\begin{tabular}{|c|c|c|c|c|c|c|}
\hline \multirow[t]{2}{*}{ Animal groups } & \multirow[b]{2}{*}{$\begin{array}{c}\text { Animal } \\
\text { numbers }\end{array}$} & \multicolumn{5}{|c|}{ Weeks Post Vaccination } \\
\hline & & 0 & 1 & 2 & 3 & 4 \\
\hline \multirow[t]{2}{*}{ Group (1) } & 1 & 0 & 2 & 16 & 64 & 128 \\
\hline & 2 & 0 & 2 & 8 & 32 & 128 \\
\hline \multirow[t]{2}{*}{ P- $0.125 \%$ Vaccine } & 3 & 0 & 2 & 8 & 32 & 64 \\
\hline & GMT & 0 & 2 & 10.6 & 42.6 & 106.6 \\
\hline \multirow[t]{2}{*}{ Group (2) } & 1 & 0 & 0 & 2 & 32 & 128 \\
\hline & 2 & 0 & 0 & 2 & 16 & 64 \\
\hline \multirow[t]{2}{*}{ P-1\%Vaccine } & 3 & 0 & 0 & 4 & 32 & 64 \\
\hline & GMT & 0 & 0 & 2.6 & 26.6 & 85.3 \\
\hline \multirow[t]{2}{*}{ Group (3) } & 1 & 0 & 8 & 32 & 128 & 128 \\
\hline & 2 & 0 & 8 & 16 & 128 & 128 \\
\hline \multirow[t]{2}{*}{ S- $0.125 \%$ Vaccine } & 3 & 0 & 16 & 32 & 64 & 128 \\
\hline & GMT & 0 & 10.6 & 26.6 & 106.6 & 128 \\
\hline \multirow[t]{2}{*}{ Group (4) } & 1 & 0 & 8 & 16 & 128 & 128 \\
\hline & 2 & 0 & 4 & 8 & 64 & 128 \\
\hline \multirow[t]{2}{*}{ CSN $1 \%$ Vaccine } & 3 & 0 & 4 & 8 & 64 & 64 \\
\hline & GMT & 0 & 5.3 & 10.6 & 85.3 & 106.6 \\
\hline \multirow[t]{2}{*}{ Group (5) } & 1 & 0 & 0 & 4 & 8 & 32 \\
\hline & 2 & 0 & 0 & 8 & 32 & 64 \\
\hline \multirow[t]{2}{*}{ (C- Vaccine) } & 3 & 0 & 0 & 4 & 16 & 64 \\
\hline & GMT & 0 & 0 & 5.3 & 18.6 & 53.3 \\
\hline Group (6) & 1 & 0 & 0 & 0 & 0 & 0 \\
\hline Unvaccinated & 2 & 0 & 0 & 0 & 0 & 0 \\
\hline \multirow[t]{2}{*}{ Animals } & 3 & 0 & 0 & 0 & 0 & 0 \\
\hline & GMT & 0 & 0 & 0 & 0 & 0 \\
\hline
\end{tabular}

PPRV- neutralizing antibody titers expressed as the reciprocal of the least serum dilution that inhibit the appearance of CPE produced by 100 TCID50/0.1 ml of PPRV on vero cells.

Group (1): Animals vaccinated with PPR vaccine based on $0.125 \%$ Calcium phosphate nanoparticles (P-0.125\% coded vaccine). Group (2): Animals vaccinated with PPR vaccine based on $1 \%$ Calcium phosphate nanoparticles (P-1\% coded vaccine). Group (3): Animals vaccinated with PPR vaccine based on $0.125 \%$ Chitosan nanoparticles (S- $0.125 \%$ coded vaccine) . Group (4): Animals vaccinated with PPR vaccine based on 1\% Chitosan nanoparticles (S-1\% coded vaccine) . Group (5): Animals vaccinated with PPRV conventional type vaccine (positive control) (C coded vaccine). Group (6): Unvaccinated control animals (negative control). 


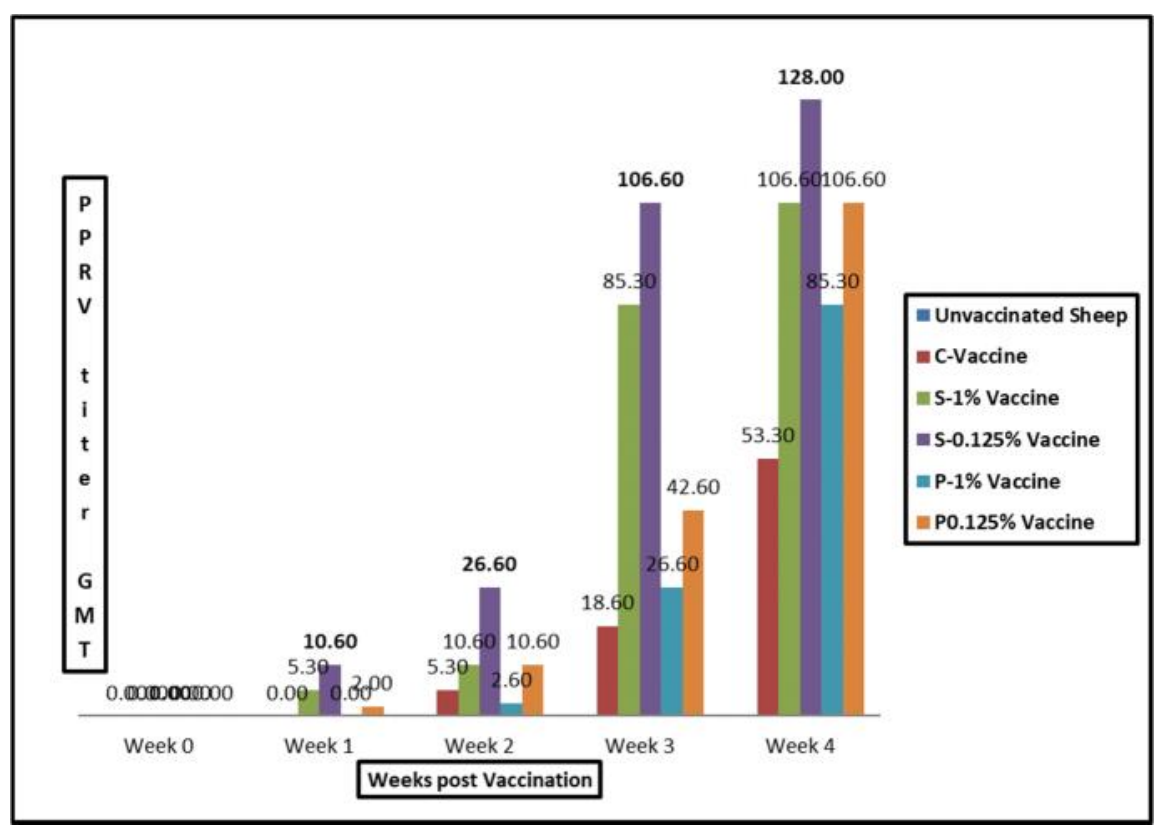

Chart (1): The level of neutralizing PPRV antibody titers in sera of sheep vaccinated with Conventional and different nanoparticles-based vaccines. MT= Geometric Mean neutralizing antibody Titers.

Table 2. Comparison between PPRV titer expressed by log 10 TCID50/ml just before and after lyophilization in four groups of nanoparticles-based vaccines with the conventional vaccine.

\begin{tabular}{ccccc}
\multicolumn{2}{c}{ PPR vaccine type } & \multicolumn{2}{c}{ PPRV titer expressed as $\log 10 \mathrm{TCID}_{50} / \mathrm{ml}$} & Titer loss \\
Group & Discription & Pre-lyophilization & Post-lyophilization & \\
No. & & & & \\
\hline 1 & P-0.125\% Vac. & 6.4 & 5.9 & 0.5 \\
2 & P- $1 \%$ Vac. & 6.3 & 5.7 & 0.6 \\
3 & S- $0.125 \%$ Vac. & 6.5 & 6.1 & 0.4 \\
4 & S- $1 \%$ Vac. & 6.3 & 5.8 & 0.5 \\
5 & C- Vaccine & 6.6 & 6.0 & 0.6 \\
\hline
\end{tabular}

Table 3. PPRV titer in vaccine groups (conventional one and nanoparticles-based) stored at $\underline{4^{\circ} \mathbf{c}}$

\begin{tabular}{|c|c|c|c|c|c|}
\hline \multirow[b]{2}{*}{ Period in weeks } & \multicolumn{5}{|c|}{ Vaccine Group number } \\
\hline & C & P-0.125\% & P-1\% & S-0.125\% & S-1\% \\
\hline Just Post lyophilization & $* 6.0$ & 5.9 & 5.7 & 6.1 & 5.8 \\
\hline Week-2 & 6.0 & 5.8 & 5.6 & 6.1 & 5.8 \\
\hline Week-4 & 6.0 & 5.8 & 5.6 & 6.0 & 5.6 \\
\hline Week-6 & 5.9 & 5.6 & 5.5 & 6.0 & 5.5 \\
\hline Week-8 & 5.7 & 5.5 & 5.4 & 5.9 & 5.5 \\
\hline Week-10 & 5.6 & 5.4 & 5.3 & 5.8 & 5.4 \\
\hline Week-12 & 5.5 & 5.4 & 5.1 & 5.7 & 5.3 \\
\hline Loss of efficacy & 0.5 & 0.5 & 0.6 & 0.4 & 0.5 \\
\hline
\end{tabular}

*PPRV titer expressed as log 10 per $1 \mathrm{ml}$ of lyophilized-reconstituted vaccine.

Group no. 1 represent the conventional vaccine (C-Vaccine).

Group no. 2 represent the vaccine prepared with CaPN 0.125\% (P-0.125\% Vaccine).

Group no. 3 represent the vaccine prepared with CaPN $1 \%$ (P-1\% Vaccine).

Group no. 4 represent the vaccine prepared with CSN $0.125 \%$ (S-0.125\% Vaccine).

Group no. 5 represent the vaccine prepared with CSN $1 \%$ (S-1\% Vaccine). 
Table 4. PPRV titer in vaccine groups (conventional one and nanoparticles-based) stored at $\underline{\mathbf{2 5}} \mathbf{c}$

\begin{tabular}{ccccccc}
\hline & \multicolumn{7}{c}{ Vaccine Group number } \\
Period in weeks & C & P-0.125\% & P-1\% & S-0.125\% & S-1\% \\
\hline Post lyophilization & $* 6.3$ & 6.0 & 5.9 & 6.1 & 6.0 \\
Week-2 & 5.8 & 5.8 & 5.8 & 5.7 & 5.5 \\
Week-4 & 5.6 & 5.8 & 5.2 & 5.4 & 5.1 \\
Week-6 & 5.3 & 5.6 & 4.5 & 5.2 & 4.7 \\
Week-8 & 5.1 & 5.5 & 4.1 & 5.0 & 4.3 \\
Week-10 & 4.6 & 5.4 & 3.9 & 4.6 & 3.9 \\
Week-12 & 4.1 & 3.9 & 3.8 & 4.2 & 3.8 \\
Loss of efficacy & 2.2 & 2.1 & 2.1 & $\mathbf{1 . 9}$ & 2.2
\end{tabular}

*PPRV titer expressed as log 10 per $1 \mathrm{ml}$ of lyophilized-reconstituted vaccine.

Group no. 1 represent the conventional vaccine (C-Vaccine).

Group no. 2 represent the vaccine prepared with CaPN $0.125 \%$ (P-0.125\% Vaccine).

Group no. 3 represent the vaccine prepared with CaPN $1 \%$ (P-1\% Vaccine).

Group no. 4 represent the vaccine prepared with CSN $0.125 \%$ (S-0.125\% Vaccine).

Group no. 5 represent the vaccine prepared with CSN $1 \%$ (S-1\% Vaccine).

Table 5. PPRV titer in vaccine groups (conventional one and nanoparticles-based) stored at $\underline{\mathbf{3 7}}{ }^{\circ} \mathbf{c}$

\begin{tabular}{cccccc}
\hline & \multicolumn{5}{c}{ Vaccine Group number } \\
Period in days & C & P-0.125\% & P-1\% & S-0.125\% & S-1\% \\
\hline Post lyophilization & $* 5.9$ & 5.8 & 6.0 & 6.1 & 6.0 \\
Day-1 & 5.6 & 5.6 & 5.4 & 5.7 & 5.5 \\
Day-3 & 5.1 & 5.1 & 4.9 & 5.2 & 5.1 \\
Day-5 & 4.9 & 4.6 & 4.6 & 5.1 & 4.7 \\
Day-7 & 4.3 & 4.3 & 4.5 & 4.7 & 4.4 \\
Loss of efficacy & 1.6 & 1.5 & 1.5 & $\mathbf{1 . 4}$ & 1.6 \\
\hline
\end{tabular}

*PPRV titer expressed as log 10 per $1 \mathrm{ml}$ of lyophilized-reconstituted vaccine.

\section{DISCUSSION}

Peste des petits ruminants appeared firstly in Egypt in January 1987, with a very high morbidities and mortalities (Ikram et al.1988). Vaccination remains the most cost-effective biomedical approach to control infectious disease in livestock using either live attenuated or inactivated vaccines (Paillot et al., 2008).

Enhancing of PPR vaccine immunogenicity considered a wide scope among vaccine producers but the major challenge that threatens the use of PPR vaccine was the need for a cold chain in order to keep the vaccine intact. Although, PPR disease is endemic in tropical areas, so creating new was for improving the keeping quality and thermostability still PPR vaccine producers challenge.
Regarding Immunogenicity test (SNT) as shown in table (1) and chart (1), PPRV neutralizing antibody titers expressed as the reciprocal of the least serum dilution that inhibit the appearance of CPE produced by 100 $\mathrm{TCID}_{50} / 0.1 \mathrm{ml}$ of PPRV on VERO cells, the following facts will be clearly noticed that, the fastest onset of appearance of the neutralizing antibody titers was in group 3 (CSN 0.125\%) with GMT=10.6 by the end of the first week. that consider protective. However, the highest results obtained by the end of 4th; week also, observed in group 3 (CSN $0.125 \%$ ) with GMT $=128$. Also, the lower the concentration of both nanoparticles vaccines, the higher the neutralizing antibody titers. Finally, all nanoparticle incorporated vaccines (group 
$1,2,3 \& 4)$ yielded too much better result rather than the conventional vaccine (group 5).

This study results are agreed with the results obtained by (Volkova et al., 2014) who stated that inactivated New Castle disease virus (NDV) vaccine in combination with $\mathrm{CSN}$ or CaPN increased the antibody titers in blood and mucosal samples of chickens was observed when compared with the administration of NDV antigen only and also observed that CSN-based vaccine has higher antigenic and protective activity than vaccine contains $\mathrm{CaPN}$ following a challenge with ND virus considering $\mathrm{CSN}$ as a potential adjuvant in poultry vaccines. Also, this study results came in agreement with (Wen et al., 2011 and Zheng et al., 2011) where they showed that when mice were inoculated subcutaneously twice with a dose of $25 \mu \mathrm{g}$ of the low immunogenic protein ovalbumin that was dissolved in saline containing CSN(12.5,50 or $200 \mu \mathrm{g}$ ), the serum OVA-specific IgG1, IgG2a, $\mathrm{IgG} 2 \mathrm{~b}$ antibody titer were enhanced. The same results were ascertained by (Greenwood et al., 2008) who managed to elevate the immune response of sheep against Foot and Mouth Disease Virus specific synthetic peptides through their combination with chitosan nanobeads resulting in a significant increase in cellular and humoral immune responses.

Referring to results in table (2) that shows a comparison between nanoparticles-based vaccine and the conventional one, before and after lyophilization. It well known that lyophilization causes loss in titer by about 0.6 $\log 10 \mathrm{TCID} 50 / \mathrm{ml}$ under optimum conditions (within the permissible limits) while it was observed that almost all nanoparticles vaccines showed lower loss than that of the conventional vaccine however, the lowest loss recorded by CSN $0.125 \%$. That may explained on the basis of protection of virus during lyophilization process needs adding a stabilizer, and one of the major nanoparticles action is stabilization of antigen as stated by
(Akagi et al., 2012, Gengoux and Leclerc 1995\& Zhao et al., 2014) so, it acts as an additional stabilizer added that means nanoparticles when augment PPR vaccine it can add an additional protection for virus and therefore can increase the stability and vaccine keeping quality.

Respecting results in table (3), regarding keeping vaccines in $\left(4^{\circ} \mathrm{c}\right)$, we can note that lowest loss of efficacy among all vaccine batches used in the trial is batch no. (4) of CSN $0.125 \%$.However, all batches showed that after 12 weeks the titer still high and considered protective and valid for use. these results was being in the midway between some scientists that confirmed that after a short period (only 30 days) the vaccine loss its validity as (Sarkar et al., 2003) while on the contrary others stated that vaccine can be valid for a period more than 12 months as Silva et al.,( 2011) or 7 months as reported by OIE 2013 where the titer remains protective (more than $2.5 \log _{10}$ ).

Regarding the obtained results in table (4 and $5)$, regarding keeping vaccines in $\left(25^{\circ} \mathrm{c}\right.$ and $37^{\circ} \mathrm{c}$ ), PPR vaccine (as the majority of live attenuated vaccines) is sensitive to elevated temperature and suffered from damage and big loss of efficacy (Kristensen et al., 2011).While the results are contradicting with that recorded by Sarkar et al., (2003) who said that, the vaccine when kept in $37^{\circ} \mathrm{c}$ will be lost just after 10 hours. Also, these results contrary to that obtained by Riyesh, (2011) who showed that the longest time of vaccine validity were about 7 days when kept in $37^{\circ} \mathrm{c}$.

\section{Conclusion}

Nanoparticles considered as an additional stabilizer for PPR vaccine that can protect the virus during the process of lyophilization and subsequently lowered the expected loss of virus titer and in turns keeps the efficacy of lyophilized vaccine in a much better way than the use of conventional stabilizers alone. CSN is more recommended for use with PPR 
vaccine preparation because of its proven immunogenicity, tolerability for animals (easily degradable), low preparation cost as well as better results in thermostability and the most recommended concentration for vaccine preparation is $0.125 \%(\mathrm{w} / \mathrm{v})$.

\section{REFERENCES}

Akagi T, Baba M, Akashi M. (2012): Biodegradlbe noanoparticles as vaccine adjuvant and delivery systems: regulation of immune responses by nanoparticlebased vaccine. In:Kunugi S, Yamaoka T, editors. Polymers in nanomedicine. Berlin Springer-Verlag Berlin; p.31-64.

CFR (1987): USA Code of Fedral Regulations.

De Campos, A.M.; Sanchez, A.; Alonso, M.J. (2001): Chitosan nanoparticles: A new vehicle for the improvement of the delivery of drugs to the ocular surface. Application to cyclosporin A. Int. J. Pharm., 224, 159-168 .

Gengoux C and Leclerc C (1995): In vivo induction of $\mathrm{CD} 4+\mathrm{T}$ cell responses by antigen covalently linked to synthetic micro-spheres does not require adjuvant; Int. Immunol.745-53.

Greenwood L.V., Deanne, Kemperly Dynon, Martha Kalkanidis, Sue Xiang, Magdalena Plebanski and Jean-Pierre Y. Scheerlinck (2008): Vaccination against foot and mouth disease virus using peptides conjugated to nano-beads. Vaccine, 26: 2706-2713.

He Q, Mitchell AR, Johnson SL, Wagner-Bartak C, Morcol T, et al. (2000): Calcium phosphate nanoparticle adjuvant. Clin Diagn Lab Immunol 7(6): 899-903.

Karim, A.I; A; El-danaf, N.A.; EL-Nakasly, S. and House, J. (1988): Isolation of a viral agent from Egyptian goats suspected to be PPR virus Infected, J. Egypt. Vet. Med. Ass., 48(3):429-435.
Illum, L.; Jabbal-Gill, I.; Hinchcliffe, M.; Fisher, A.N.; Davis, S.S., (2001): Chitosan as a novel nasal delivery system for vaccines. Adv. Drug Deliv. Rev., 51, 81-96 .

Joyappa, D.H., C. Ashok Kumar, N. Banumathi, Golla Rama Reddy and Veluvarthy V.S. Suryanarayana, (2009): Calcium phosphate nanoparticle prepared with foot and mouth disease virus P1-3CD gene construct protects mice and guinea pigs against the challenge virus Veterinary Microbiology, 139: 58-66.

Kristensen D., Chen D., Cummings R. (2011): Vaccine stabilization: research, commercialization and potential impact. Vaccine, 29 (41): 7122-7124.

Mody KT, Popat A, Mahony D, Cavallaro AS, Yu C, Mitter N. (2013): Mesoporous silica nanoparticles as antigen carriers and adjuvants for vaccine delivery. Nanoscale; 5:5167-79.

Nordly, P., H.B. Madsen, H.M. Nielsen and C. Foged, (2009): Status and future prospects of lipid-based particulate delivery systems as vaccine adjuvants and their combination with immunostimulators. Expert Opinion on Drug Delivery, 6: 657672.

OIE (2013): Manual of standards for diagnostic tests and vaccines.

Paillot, R; Case, R; Ross, J.; Newton, R .and Nugent, J. (2008): Equine Herpes virus1: Virus, Immunity and Vaccines. The open veterinary Science Journal, 2, 68-91.

Riyesh, T., Balamurugan, V., Sen, A., Bhanuprakash, V. Venkatesan, G., Yadav, V., Singh, R. (2011): Evaluation of efficacy of stabilizers on the thermostability of live attenuated thermoadapted peste des petits ruminants vaccines. Virologica Sinica, 26 (5): 324337 
Sarkar J.; Sreenivasa, B.P.; Singh, R.P. Dhar, P and Andyopadhyay, S.K., (2003): Comparative efficacy of various chemical stabilizers on the thermostability of a live attenuated peste des petits ruminants (PPR) vaccine. Vaccine 21 (2003): 47284735 .

Shao-zhi Z, Huan Q, Zhen W, Ju-li F, Qian Z, Guang-ming C, Rui L, Shan F and Jie S. (2010): Preliminary study on the freezedrying of human bone marrow-derived mesenchymal stem cells. J. Zhejiang Univ. Sci. Bv. 11.(11)

S. Jain, D.T. O'Hagan, and M. Singh, (2011): The long-term potential of biodegradable poly (lactideco-glycolide) microparticles as thenext-generation vaccine adjuvant. 1731-1742.

Silva, A.C., Carrondoa, M.J.T., Alves, P.M. (2011): Strategies for improved stability of Peste des Petits Ruminants Vaccine. Vaccine 29:4983-4991

Van der Lubben, I.M.; Verhoef, J.C.; Borchard, G.; Junginger, H.E. (2001): Chitosan microparticles for oral vaccination: Preparation, characterization and preliminary in vivo uptake studies in murine Peyer's patches. Biomaterials, 22, 687-694 .

Volkova, M.A., Irza, A.V., Chvala, I.A., FrolovS. F., Drygin, V.V. and Kapczynski, D. (2014): Adjuvant effects of chitosan and calcium phosphate particles in an inactivated Newcastle disease vaccine. Avian Dis. 58(1):46-52.

Wang J and Zhang H. (2007): The influence of one-step $-80{ }^{\circ} \mathrm{C}$ cryopreservation on the osteogenesis differentiation ability of human bone marrow-derived mesenchymal stem cells. Guangdong Med., 28(3), 365- 367.

Wen, Z.S., Xu, Y.L., Zou, X.T. and Xu, Z.R. (2011): Chitosan Nanoparticles act as an adjuvant to promote both Th1 and Th2 Immune response induced by ovalbumin in mice. Marine drugs, 9(6),1038-1055.

Y. Fujita, and H. Taguchi, (2011): Current status of multiple antigens presenting peptide vaccine systems: Application of organic and inorganic nanoparticles. Chemistry Central Journal 5, 1-8.

Zhao, L.; Seth, A.; Wibowo, N.; Zahao, C.; Mitter, N.; Yu, C. and Middelberg, A. (2014): Nanoparticles vaccines, Elsevier; 32:327-337.

Zheng-Shun Wen, Ying-Lei Xu, Xiao-Ting Zou and Zi-Rong Xu. (2011): Chitosan Nanoparticles Act as an Adjuvant to Promote both Th1 and Th2 Immune Responses Induced by Ovalbumin in Mice. Marine Drugs, 9, 1038-1055.

Zhou XL, Zhu H, Zhang SZ, Zhu FM, Chen GM and Yan LX. (2007): Freeze-drying of human platelets: influence of saccharide, freezing rate and cell concentration. Cryo Letters., 28(3), 187-196.

Zhu, B.; Qie, Y.; Wang, J.; Zhang, Y.; Wang, Q.; $\mathrm{Xu}$, Y.; Wang, H. (2007): Chitosan microspheres enhance the immunogenicity of an Ag85B-based fusion protein containing multiple T-cell epitopes of Mycobacterium tuberculosis. Eur. J. Pharm. Biopharm., 66, 318-326 . 